\title{
Managing Insecticide Resistance by Mass Release of Engineered Insects
}

\author{
NINA ALPHEY, ${ }^{1,2,3}$ PAUL G. COLEMAN, ${ }^{2,3,4}$ CHRISTL A. DONNELLY, ${ }^{5}$ aNd LUKE ALPHEY ${ }^{1,2,6}$
}

\begin{abstract}
J. Econ. Entomol. 100(5): 1642-1649 (2007)
ABSTRACT Transgenic crops producing insecticidal toxins are now widely used to control insect pests. The benefits of this method would be lost if resistance to the toxins spread to a significant proportion of the pest population. The primary resistance management method, mandatory in the United States, is the high-dose/refuge strategy, requiring toxin-free crops as refuges near the insecticidal crops, and the use of toxin doses sufficiently high to kill insects heterozygous for a resistance allele, thereby rendering resistance functionally recessive. We propose that mass-release of harmless susceptible (toxin-sensitive) insects could substantially delay or even reverse the spread of resistance. Mass-release of such insects is an integral part of release of insects carrying a dominant lethal (RIDL), a method of pest control related to the sterile insect technique. We show by mathematical modeling that specific RIDL strategies could form an effective component of a resistance management strategy for plant-incorporated protectants and other toxins.
\end{abstract}

KEY WORDS resistance management, Bacillus thuringiensis, mathematical modeling, RIDL, highdose/refuge strategy

Transgenic plants, principally maize, Zea mays L., and cotton, Gossypium hirsutum L., producing insecticidal proteins derived from Bacillus thuringiensis Berliner (Bt), grew on 32 million ha worldwide during 2006 (James 2006). The use of such plant-incorporated protectants (PIPs) can reduce reliance on insecticidal sprays, thereby providing economic, health, and environmental benefits (Shelton et al. 2002). However, such benefits would be reduced or even eliminated if resistance to PIPs became widespread in pest populations. For many pest species, strains substantially resistant to $\mathrm{Bt}$ toxins have been selected in the laboratory, although resistance to $\mathrm{Bt}$ crops has not yet been found in the field (Tabashnik et al. 2003, 2005; Bates et al. 2005; Ferry et al. 2006). However, field resistance to Bt sprays has been found in diamondback moth, Plutella xylostella (L.) (Ferré and Van Rie 2002), and greenhouse populations of cabbage looper, Trichoplusia ni (Hübner) (Janmaat and Myers 2003). Therefore, considerable attention has been devoted to methods to prevent or reverse the spread of resistance through wild pest populations.

\footnotetext{
${ }^{1}$ Department of Zoology, University of Oxford, South Parks Rd., Oxford OX1 3PS, United Kingdom.

${ }^{2}$ Oxitec Limited, 71 Milton Park, Oxford OX14 4RX, United Kingdom.

${ }^{3}$ These authors contributed equally to this work.

${ }^{4}$ Department of Infectious \& Tropical Diseases, London School of Hygiene \& Tropical Medicine, Keppel St., London WC1E 7HT, United Kingdom.

${ }^{5}$ Department of Infectious Disease Epidemiology, Imperial College London, Faculty of Medicine, Norfolk Place, London, W2 1PG, United Kingdom.

${ }^{6}$ Corresponding author, e-mail: luke.alphey@zoo.ox.ac.uk.
}

Mathematical models predict that the high-dose/ refuge strategy slows or prevents the spread of resistance (Alstad and Andow 1995; Gould 1998; Carrière and Tabashnik 2001; Tabashnik et al. 2003, 2004, 2005; Vacher et al. 2003). Resistance to Bt toxins is thought to be partially dominant, with a strong recessive component (Ferré and Van Rie 2002, Tabashnik et al. 2003). The high-dose part of the strategy aims to reduce this to purely recessive resistance by using sufficiently high doses of the toxin that heterozygotes are not functionally resistant (i.e., do not survive). Refuges are a crucial component; they provide an area of non-Bt host plants and thereby provide a source of susceptible (toxin-sensitive) genotypes that, by mating with insects from toxin-treated regions, tend to reduce the frequency of resistance alleles.

In principle, an alternate source of susceptibility alleles could be provided by mass-rearing susceptible insects and deliberately releasing them into the environment. We first investigated the simple case of the release of wild-type, susceptible insects, before exploring the potential use of more sophisticated, nonwild-type strains.

The mass release of normal, fertile pest insects is unlikely to be acceptable in practice. In the sterile insect technique (SIT), pest insects are indeed massreared and released, but only after they have been sterilized by irradiation (Knipling 1955, Krafsur 1998, Dyck et al. 2005). In this case, no viable hybrids are formed between the released insects and the wild population, so there is no introgression of susceptibility alleles into the wild population. However, we have previously proposed a modification to the SIT in which the released insects are not irradiated, but instead are 


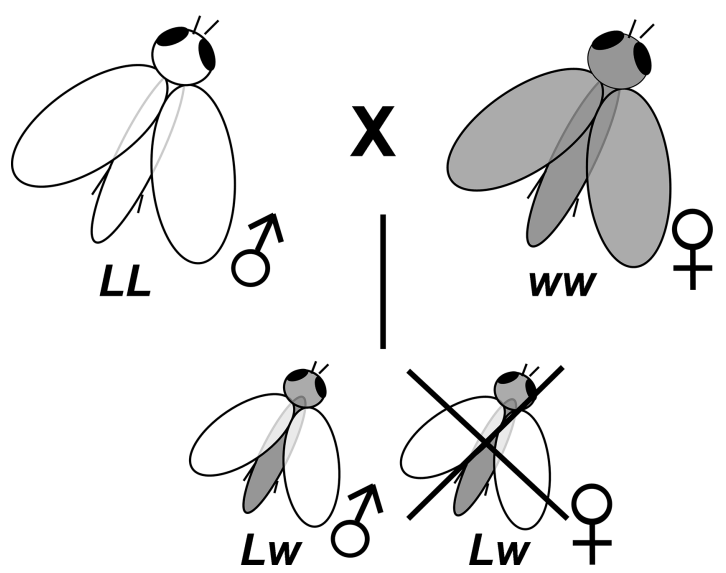

Fig. 1. Female-lethal RIDL. Released RIDL males are homozygous for one or more female-specific dominant lethal genes $(L L)$. Offspring of RIDL males and wild females $(w w)$ are heterozygous for the RIDL construct $(L w)$. $L w$ females die but $L w$ males are unaffected, inheriting one copy to pass on to half their offspring.

homozygous for one or more dominant lethal genes (release of insects carrying a dominant lethal [RIDL]) (Thomas et al. 2000, Alphey 2002, Alphey and Andreasen 2002, Gong et al. 2005, Alphey et al. 2007). One version of this system posits the release of male insects homozygous for one or more female-specific dominant lethals (RIDL males) (Fig. 1). These lethal genes would be repressed throughout mass rearing, except in the generation intended for release (maleonly release is generally desirable for SIT; Rendon et al. 2004, Marec et al. 2005). Released RIDL males would mate with wild females; all of their $\mathrm{F}_{1}$ progeny would inherit a dominant female-specific lethal. The $\mathrm{F}_{1}$ females would die, thereby reducing the reproductive potential of the wild population (much like the SIT), but the males would live. Half of their $\left(F_{2}\right)$ daughters would also inherit the RIDL gene(s) and die, so there would be some modest additional suppression. Strains with the necessary genetic properties for this female-lethal version of RIDL have been constructed in several species, with proof-of-principle in Drosophila melanogaster (Meigen) (Heinrich and Scott 2000, Thomas et al. 2000) and recent success in a major pest insect, the Mediterranean fruit fly, Ceratitis capitata (Wiedemann) (Fu et al. 2007). No such strains have yet been described in Lepidoptera, but there is active work and progress in this area.

The potential utility of such systems for control of target pest populations has been explored by mathematical modeling, which indicated several advantages over conventional SIT (Schliekelman and Gould 2000, Thomas et al. 2000, Alphey 2002, Alphey and Andreasen 2002). A previously unidentified and novel feature of such systems is that mating between released males and wild females will produce viable, fertile male progeny, leading to introgression of alleles from the mass-reared strain into the wild population. This has significant benefits for any control program combining the use of female-specific RIDL with the use of toxins, such as PIPs.

The high-dose/refuge strategy is underpinned by a body of theoretical analysis and some years of field experience (Alstad and Andow 1995; Gould 1998; Carrière and Tabashnik 2001; Shelton et al. 2002; Glaser and Matten 2003; Tabashnik et al. 2003, 2005; Bates et al. 2005). Assuming random mating and completely (or nearly) recessive resistance, the factors favoring prevention or reversal of resistance are thought to be nonrecessive costs of resistance, low initial frequency of the resistance allele $(r)$, large refuges, and incomplete resistance $(r r$ genotype fitness on transgenic crops less than on nontransgenics). We explored the effects of the release of RIDL males on the spread of resistance, and we investigated the relationship between the ratio of released RIDL males to males in the wild, the refuge size and the fitness parameters of the resistant insects.

\section{Materials and Methods}

Use of Wild-Type Insects. We used a deterministic, discrete-generation population genetic model (Carrière and Tabashnik 2001, Tabashnik et al. 2005) and assume a closed homogeneous population, with random mating, and no immigration, emigration, or mutation. We assume that eggs are male and female in equal proportions (sex ratio 1:1). Larvae, the Bt-susceptible life stage, are assumed to spend their whole development either on $\mathrm{Bt}$ crops or in the refuge; migration occurs after emergence as adults and before mating.

We assume a fixed crop population, a proportion $\Phi$ of which expresses Bt toxins, so the conventional (non-Bt) crop refuge size is $1-\Phi$. Larval susceptibility to Bt toxins is assumed to be controlled by a single autosomal locus with two alternative alleles: resistant $r$ (frequency $p$ ) and susceptible $s$ (frequency $q, p+q=1)$. There are three genotypes at this locus: $s s, s r$, and $r r$.

All insects are affected by Bt toxins. Resistant insects are expected to be at a selective disadvantage on non-Bt host plants. We assume that all fitness costs associated with the $s$ and $r$ alleles take effect during the larval stage. Assuming that larvae are distributed at random across $\mathrm{Bt}$ and non-Bt crops, the average relative fitness of larvae of genotype $i$ (i: ss, sr, or $r r)$ is as follows:

$$
\Omega_{i}=\omega_{i} \Phi+v_{i}(1-\Phi)
$$

where $\omega_{i}$ is the relative fitness of larvae on Bt crops, and $v_{i}$ is relative fitness of larvae in non-Bt crops. Fitness is measured relative to ss homozygotes on non-Bt crops (so $v_{s s}=1$ ).

With no release of insects, the change in $r$ allele frequency in the next generation is given by

$$
\Delta p=\frac{p q\left(p \Omega_{r r}+(q-p) \Omega_{s r}-q \Omega_{s s}\right)}{q^{2} \Omega_{s s}+2 p q \Omega_{s r}+p^{2} \Omega_{r r}}
$$

With release of homozygote susceptible adults ( $s s$ wild-type males and females in equal numbers) in ratio $d: 1$ to adults in the wild, the change in $r$ allele frequency is given by 
$\Delta p=\frac{p\left(p q \Omega_{r r}+(q+d)(q-p) \Omega_{s r}-(q+d)^{2} \Omega_{s s}\right)}{(q+d)^{2} \Omega_{s s}+2 p(q+d) \Omega_{s r}+p^{2} \Omega_{r r}}$

Use of Engineered Insects. We extended the basic model to incorporate the effects of release of RIDL males. We assume that at each generation, as adults emerge before mating, mass-reared adult RIDL males are released at a fixed ratio to the number of males in the wild population. The RIDL release ratio is defined as the number of RIDL males released per insect generation, as a proportion of the number of males in the wild (of any genotype) in that generation that survived to reproductive maturity. Females mate with wild or released males at random.

All male, engineered released males are assumed to be homozygous susceptible to Bt toxins (ss) and homozygous for the RIDL construct. There are two possible alleles at the insertion site of the RIDL construct: $L$, representing insertion of the construct, and the wild-type allele $w$, representing absence of the construct. We assume the RIDL insertion and the locus controlling resistance to $\mathrm{Bt}$ are not linked and neither of these is sex-linked.

We assume the RIDL construct imposes a sex-specific fitness cost $\varepsilon$ during the larval stage. The average relative fitness of larvae of genotype $i$ is then

$$
\Omega_{i}=\left(1-\varepsilon_{i}\right)\left[\omega_{i} \Phi+v_{i}(1-\Phi)\right]
$$

where $\varepsilon_{i}$ is the sex-specific, relative fitness cost imposed by RIDL.

A simulation approach was adopted (Fig. 2) because the resulting system of difference equations is unwieldy (they involve eighteen potential genotypes and cannot be reduced to expressions in terms of $p$ ), and there are no analytical solutions for allele or genotype frequencies or for the critical release ratios or critical refuge sizes.

We assume RIDL-induced lethality is fully dominant and penetrant, so for $L w$ females $\varepsilon_{\text {female }}=1$ and average fitness $\Omega_{i}=0$, irrespective of $\Phi, \omega_{I}$ and $v_{i}$. Under these conditions, $L L$ homozygotes do not arise in the field, and there are seven male and three female viable genotypes defined at the $L / w$ autosome and $s / r$ locus. The LLss male genotype is the engineered RIDL release male, and six male genotypes (Lwss, Lwsr, Lwrr, wwss, wwsr, and wwrr) and three female genotypes (wwss, wwsr, and wwrr) arise from matings in the field. In these simulations, $\varepsilon_{\text {male }}$ was set to 0.1 for $L w$ male larvae, to allow for the modest fitness penalty likely to be associated with the RIDL insertion (for example, due to insertional mutagenesis and/or expression of the marker gene; Moreira et al. 2004, Marrelli et al. 2006).

We explored the effects of release of homozygous RIDL males on the spread of resistance by simulating the frequencies of all genotypes under the abovementioned assumptions over 200 generations. We simulated outcomes for different combinations of $v$ and $\omega$ representing fully recessive costs and benefits of resistance $\left(\omega_{r r}=0.1,0.2\right.$, or $0.4, v_{r r}=0.7$ or $0.4, v_{s r}=v_{s s}=$

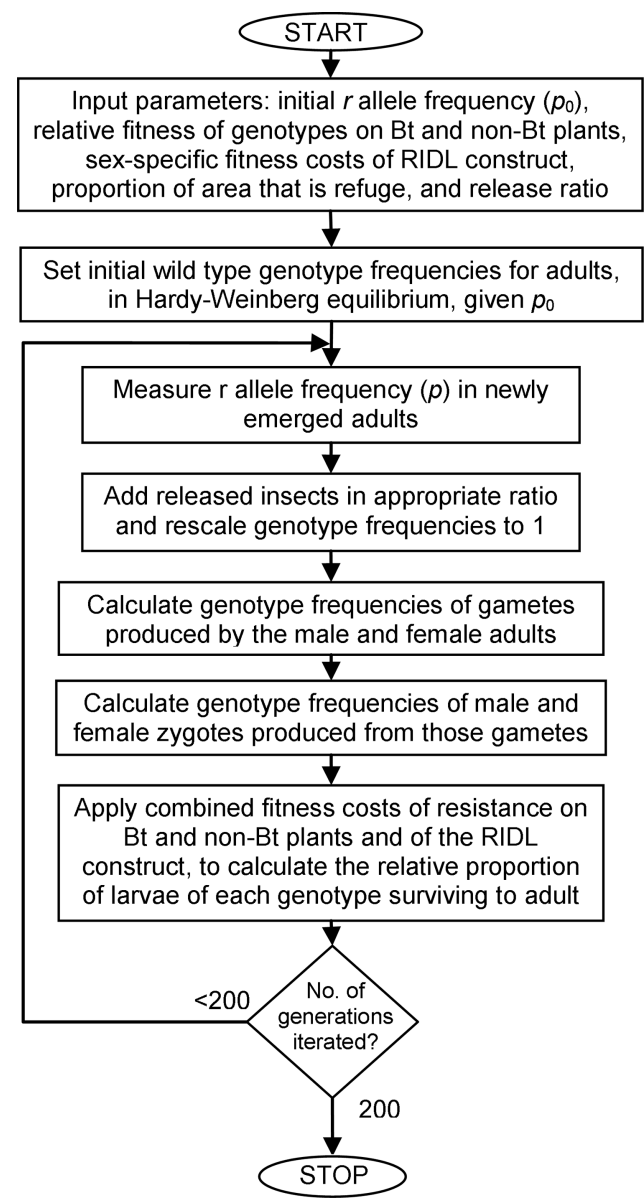

Fig. 2. Simulation.

1 , and $\left.\omega_{s s}=\omega_{s r}=0\right)$ (Carrière and Tabashnik 2001), i.e., six different combinations of $\omega_{r r}$ and $v_{r r}$, over a range of refuge sizes $(0-0.6)$ and initial $r$ allele frequencies $p_{0}(0.1,0.01$, or 0.001$)$. The range of $p_{0}$ values used reflects field data: 0.0015 estimated for tobacco budworm, Heliothis virescens (F.) (Gould et al. 1997), 0.13 and 0.16 in 1997, and a mean of 0.004 across 1998-2004 (95\% CL $=0-0.01$, annual estimates vary approximately from 0 to 0.08 ) for pink bollworm, Pectinophora gossypiella (Saunders), in Arizona (Tabashnik et al. 2000, 2005), 0.0015 (95\% credibility interval $=$ 0-0.0044) for European corn borer, Ostrinia nubilalis (Hübner), in the southern corn belt in 2000-2003 (Stodola et al. 2006), and $0.0023(95 \%$ CI $=0.0003-$ 0.0064) for sugarcane borer, Diatraea saccharalis (F.), in Louisiana in 2004 (Huang et al. 2007). We explored release ratios from 1:1,000 (one RIDL male per thousand males in the wild) to 100:1.

\section{Results}

Use of Wild-Type Insects. The average relative fitnesses of each genotype, determined from the parameters, dictate whether resistance spreads and threshold conditions for decrease of $r$ allele frequency can be 


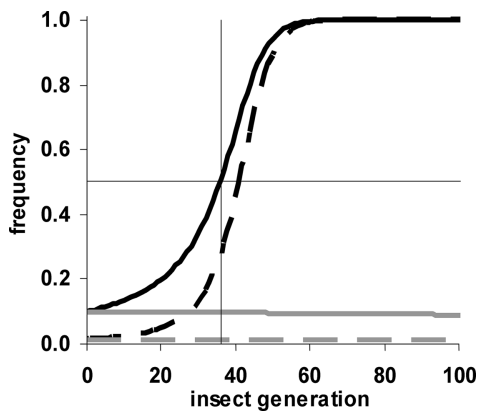

Fig. 3. Releasing RIDL males can reverse the spread of resistance. The graph shows the frequency over time of the $r$ allele (solid lines) and the $r r$ resistant phenotype (dashed lines), for no release of insects (black lines) and for a RIDL release ratio of 1:5, i.e., one RIDL male per five males in the wild (gray lines). The initial $r$ allele frequency was 0.1 , and the refuge size was $10 \%$. The costs and benefits of resistance were fully recessive $\left(v_{s r}=v_{s s}=1\right)$, with resistant homozygotes having relative fitness of 0.1 on Bt crops $\left(\omega_{r r}\right)$ and 0.4 fitness on non-Bt crops $\left(v_{r r}\right)$. Other genotypes are fully susceptible to the Bt toxins $\left(\omega_{s s}=\omega_{s r}=0\right)$. With these parameters the resistance allele frequency $p$ increases over time, reaching 0.5 in 36 generations (Carrière and Tabashnik 2001). However, release of RIDL males at a ratio of only 1:5 was sufficient to reverse this.

deduced. We found that if $s s$ homozygotes are released each generation at a constant ratio to the wild population, these thresholds increase, allowing for the same level of control to be provided by a smaller refuge.

Use of Engineered Insects. We found that, in all cases, release of RIDL males slowed the spread of
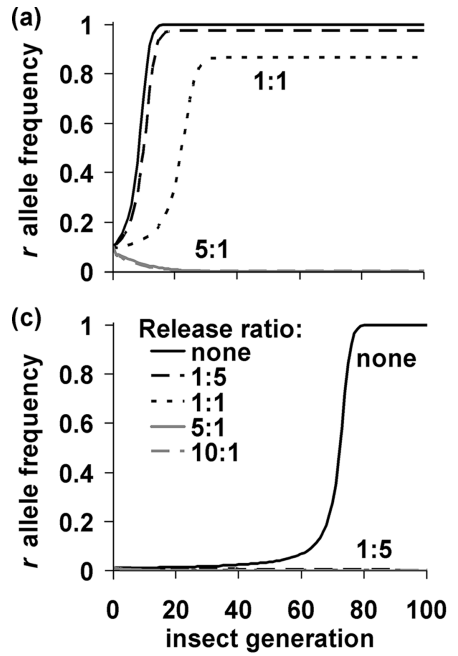

Fig. 4. Effect of release ratio on the spread of resistance, for different refuge sizes and initial allele frequencies. The graphs show the frequency over time of the $r$ allele for no release and for RIDL release ratios 1:5, 1:1, 5:1, 10:1 (a) and 11:1 (b). Fitness assumptions are as in Fig. $3\left(v_{s r}=v_{s s}=1\right.$ and $\left.\omega_{s s}=\omega_{s r}=0\right)$ except those of resistant homozygotes were 0.2 on Bt crops $\left(\omega_{r r}=\right.$ $0.2)$ and 0.7 on non-Bt plants $\left(v_{r r}=0.7\right)$. The initial $r$ allele frequency $\left(p_{0}\right)$ was $0.1(\mathrm{a}$ and b) or $0.01(\mathrm{c}$ and $\mathrm{d})$, and the refuge size was $10 \%$ ( $a$ and c) or $5 \%$ (b and d). In all cases, release slows the spread of the $r$ allele. Above a critical threshold release ratio the release reverses the spread of resistance. Below this threshold, the $r$ allele increases to an equilibrium frequency which may be $<1$. Note the potential for a long lag phase followed by rapid increase of $r$ allele frequency, where there is no, or insufficient, RIDL release.

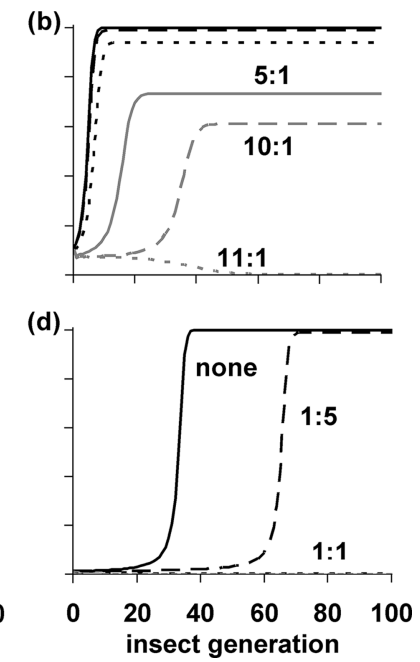
programs providing effective suppression of the target population. Figure 3 shows an example, in which $p$ (the frequency of the $r$ allele) reaches 0.5 in 36 generations with no release of insects (Carrière and Tabashnik 2001). Release of RIDL insects, at a ratio of only 1:5, reverses the spread of resistance. Figure 3 also illustrates the potential for rapid increase in the frequency of resistant individuals ( $r r$ homozygotes) after a long lag phase. This is a consequence of the nonlinear relationship between the frequency of the resistance allele $(p)$ and the frequency of resistant individuals.

We explored the effect of the RIDL release ratio on the change over time of the frequency of the resistance allele, under various assumptions about the fitness of $r r$ homozygotes. This is illustrated in Fig. 4 for a particular set of fitness values. In each case, sufficient release of RIDL males can prevent the spread of the resistance allele. Reducing the refuge size increases the rate at which the resistance allele will spread, and correspondingly increases the number of RIDL males required to be released to prevent the spread of resistance (Fig. 4, compare a and $\mathrm{c}$ with $\mathrm{b}$ and $\mathrm{d}$, respectively), although any RIDL release will always slow the spread relative to no release. As well as refuge size, another critical parameter is $p_{0}$, the initial allele frequency of $r$. For fully recessive resistance, the fitness advantage of the resistance allele is strongly frequency-dependent, as the frequency of the homozygote, the only form to gain significant advantage, is approximately the square of the allele frequency; with no RIDL release, at equilibrium, this is $p^{2}$. For given cases reversing the spread of resistance, at release 

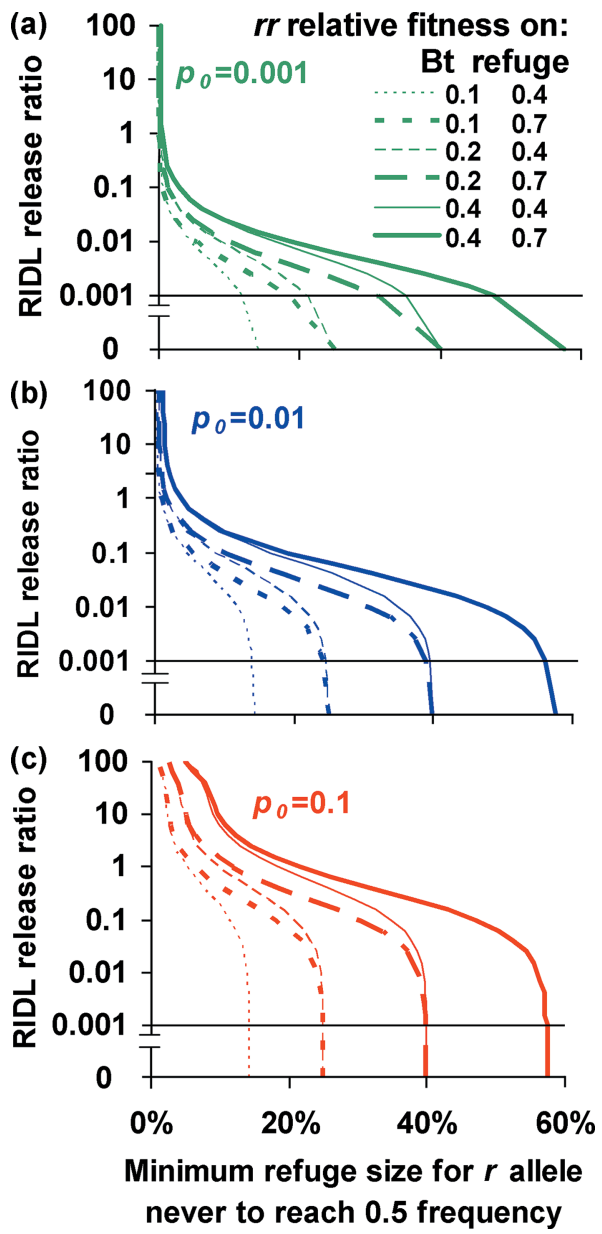

Fig. 5. Effect of RIDL release on the minimum refuge size needed to stop the spread of resistance. Critical refuge sizes at which $p$ neither increases nor decreases were calculated for the "no release" baseline case, and they were closely approximated for a range of RIDL release ratios from 1:1000 to $100: 1$ by determining the minimum refuge size for which resistance allele frequency will not reach 0.5 (i.e., will either decrease or reach a nonzero equilibrium frequency below 0.5). These are shown for three initial $r$ allele frequencies $\left(p_{0}\right): 0.001(\mathrm{a}), 0.01(\mathrm{~b})$, and $0.1(\mathrm{c})$ and for various fitness parameters. The RIDL release ratio axis is on a log scale except that the values for 0 (no release) are plotted and joined to those for 0.001 (1:1000) by straight lines. As in Figs. 3 and 4 , resistance and costs of resistance are fully recessive. Fitness of resistant homozygotes on Bt crops $\left(\omega_{r r}\right)$ is $0.1,0.2$, or 0.4 and fitness on non-Bt crops $\left(v_{r r}\right)$ is 0.4 or 0.7 , as indicated in the key in a, which applies to all panels.

fitness parameters of $r$, a higher value of $p_{0}$ (Fig. 4, compare $\mathrm{a}$ and $\mathrm{b}$ with $\mathrm{c}$ and $\mathrm{d}$, respectively) or a smaller refuge mean that a higher RIDL release ratio is required to prevent the resistance allele from spreading.

We investigated the relationship between the minimum refuge size required to prevent the spread of resistance and the RIDL release ratio, for various fitness parameters of $r$ and initial allele frequencies of $r$ (Fig. 5).

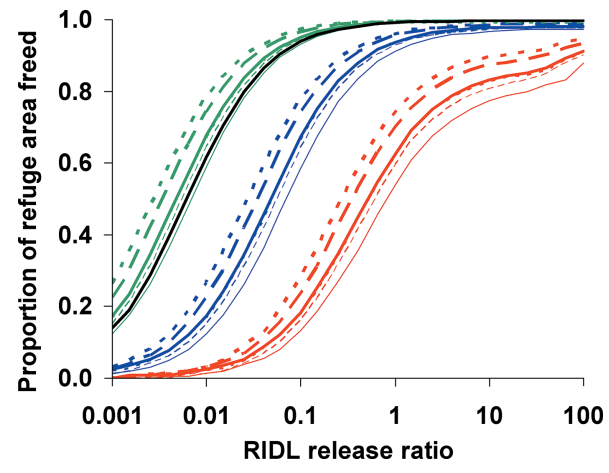

Fig. 6. RIDL release reduces the minimum refuge size needed to stop the spread of resistance. We calculated the reduction in minimum refuge size needed to stop the spread of resistance as a proportion of the equivalent minimum refuge size with no release. This proportion of refuge potentially freed to be planted with $\mathrm{Bt}$ crops is shown against RIDL release ratio for the same values of $p_{0}$ and combinations of resistant homozygote fitness as in Fig. 5. Line colors indicate values of $p_{0}$ : green, 0.001 ; blue, 0.01 ; and red, 0.1, as in Fig. $5 \mathrm{a}-\mathrm{c}$. Fitness of resistant homozygotes on Bt crops $\left(\omega_{r r}\right)$ are $0.1,0.2$, or 0.4 and on non-Bt plants $\left(v_{r r}\right)$ are 0.4 or 0.7 , as indicated in the key in Fig. 5a. In addition, the black line shows the outcomes for a rare resistance allele $\left(p_{0}=0.001\right)$ with higher homozygote fitness values: 0.8 on Bt crops and 0.9 on non-Bt plants. Although these fitness values are much higher, it nonetheless falls within the range of other outcomes with the same $p_{0}$ (green lines).

For all parameter values examined, a considerable reduction in the minimum refuge size required to prevent spread can be achieved by release of RIDL males.

Regulators have to establish advisory or mandatory refuge sizes before the fitness parameters of hypothetical resistance alleles are known. We therefore examined the equivalence between refuge size and RIDL release ratio in terms of the trade-off between refuge size and release ratio to achieve an equivalent outcome. We calculated the proportional reduction in refuge size that could be made, at a given release ratio, while still just preventing the spread of a given resistance allele (Fig. 6). The initial allele frequency $\left(p_{0}\right)$ seems critical, whereas the precise values of $v_{r r}$ and $\omega_{r r}$ have relatively little effect, even where they are close to one (e.g., black line in Fig. 6).

\section{Discussion}

We have explored the effects of the release of RIDL male insects and predict that such release would slow or reverse the spread of resistance to PIPs. Our investigation of the relationship between the ratio of released RIDL males to males in the wild, the refuge size and the fitness parameters of the resistant insects showed that RIDL release could achieve a considerable reduction in the minimum refuge size required to prevent the spread of resistance. Current SIT programs use release rates at the upper end of the range we modeled; in many cases we found that even much lower release rates would be sufficient to justify sub- 
stantial reductions in refuge size (Fig. 6). This suggests that the necessary release rates would be feasible in many cases.

For conventional SIT against a density-independent population, with no immigration or emigration, the minimum required release ratio of fully competitive males efficiently dispersed into the population is $\left(R_{0}-1\right): 1$, where $R_{0}$ is the average number of progeny produced per adult pest insect that survive to adulthood (Knipling 1955). Above this threshold ratio, the population will tend to decline, whereas at lower release ratios the population will increase, albeit more slowly than it would otherwise have done. $R_{0}$ might range from 2 to $>10$ for typical agricultural pests under field conditions. Actual release ratios used are higher, at least 10:1 and often 60:1 or higher (Bloem et al. $2005)$; this is to compensate for the reduced lifespan and competitive mating ability of mass-reared, irradiated insects, for imperfect distribution, and to achieve good suppression of the pest population, rather than just marginal control. Release ratios in these models are not exactly equivalent to the release ratios typical of SIT-based population control programs, because we do not account for reduced competitiveness and imperfect distribution. However, in a successful SIT program the release rate must be equivalent to rates of 1:1-10:1 or higher in our models, based on the $\left(R_{0}-\right.$ 1):1 argument described above. Our models therefore demonstrate that useful resistance management can be achieved at release ratios equivalent to, or lower than, those currently used for SIT (Fig. 6), especially at low $p_{0}$.

For $p_{0}=0.001$, approximately as estimated for tobacco budworm (Gould et al. 1997) and European corn borer (Stodola et al. 2006), a release ratio as low as 1:4 could rationally justify a reduction in refuge size to as low as $2-3 \%$ of the value required without RIDL release (Fig. 6). In many cases, such a low refuge size of perhaps $0.2-0.3 \%$ of the crop area would automatically be provided by alternative host plants or less than universal planting of the insecticidal variety. Reducing the size of the refuge, while maintaining equivalent resistance management, would allow higher average crop yields and more efficient use of valuable land.

It is reassuring that the proportional reductions in minimum refuge sizes are not very sensitive to differing relative fitness estimates (Fig. 6), because empirical methods for estimating these in field populations are problematic, and estimated values vary considerably (Bourguet et al. 2000; Tabashnik et al. 2004, 2005). A higher $p_{0}$ requires either a higher RIDL release ratio or a smaller reduction in the minimum refuge size to prevent resistance spreading. This sensitivity to $p_{0}$ indicates a potential advantage to implementing such a release strategy as a preventative measure, preferably before resistance reaches a detectable level, rather than as a reactive measure once resistance has reached relatively high levels.

We have focused on the idea of using engineered insects to manage resistance to Bt crops and identified several key features of the potential effect on resis- tance evolution of using such a strategy. To do so we used a population genetics approach. A population dynamics approach would be needed in addition, to assess the effect of RIDL release on the size of the pest population, and therefore on crop damage. Male release acts by substituting released males for some of the wild males with which females would otherwise have mated. Assuming that reproduction is not limited by the availability of males, no greater numbers of progeny arise and survive than would otherwise have done so, and the female-lethal effect of the RIDL construct would therefore be expected to have a population suppression effect synergistic with that provided by the Bt crops. Empirical (Carrière et al. 2003) and modeling studies (Peck et al. 1999, Caprio 2001, Sisterson et al. 2004) have shown that population sizes of susceptible insects are greatly reduced by the use of Bt crops; the additional suppression provided by the RIDL strategy should at least to some extent improve this and might in some cases result in local extinction of the pest.

Our analysis suggests that the release of femalelethal RIDL male insects could allow a very significant reduction in refuge size while maintaining equivalent resistance management. The economic attractiveness of this approach would depend on the costs of such a release program compared with the value of increased yields to be obtained by replacing refuge plants with Bt crops (net of the premium on Bt seeds). An assessment of relative cost would require an extension of our model to include population dynamics as well as genetics, because the number of insects released each generation, and therefore the cost of the program, varies with the level of population suppression.

Our analysis has primarily focused on RIDL release and refuge strategies aimed at preventing the spread of resistance from a relatively low initial level. An additional advantage of the RIDL strategy stems from the effect that increasing release ratios above the critical threshold speeds the reversal of resistance. A RIDL release program could be deployed as remedial action to counter a resistance event, by using higher release ratios more typical of SIT population suppression programs to achieve a much more rapid reduction in resistance allele frequency than would be possible using only refuges (even under a complete ban on $\mathrm{Bt}$ crops, i.e., $100 \%$ refuge, the rate of recovery would otherwise be constrained by the fitness cost of resistant insects on non-Bt plants).

Given that SIT is already operated on an industrial and areawide scale against pink bollworm and codling moth, Cydia pomonella (L.) (Dyck et al. 2005), rearing and distributing lepidopteran pests on a suitable scale is feasible. If the relatively low numbers that would be required for routine use in resistance management programs could be produced from spare capacity in existing facilities, the marginal cost of such programs would be smaller than if dedicated facilities were needed.

This model is not sufficiently sophisticated alone to determine an appropriate release schedule. In any case, as the numbers of males released in the model are 
proportional to the numbers of males in the wild each generation, some estimation would be required. Population sizes would generally be small, but if resistance did evolve this would tend to increase the population size (Peck et al. 1999, Sisterson et al. 2004), and consequently the numbers of RIDL males to be released. For implementation purposes, we would envisage starting with a prudent approximation for release numbers, based on survey data and any available estimates of resistance allele frequency, and using sample data over time to obtain estimates of the numbers of homozygous RIDL males relative to males of other genotypes and adjust the release rate accordingly.

One of the attractive features of this method for managing the spread of resistance is that no prior knowledge of the mechanism of resistance is required. Therefore, this approach provides, in principle, a new and general method for managing the spread of pest resistance to toxins such as engineered insecticidal crop plants.

\section{Acknowledgments}

We thank Mike Bonsall and Krisztián Mágori for advice, encouragement, and review of the manuscript. This work was supported by the UK Biotechnology and Biological Sciences Research Council.

\section{References Cited}

Alphey, L. 2002. Re-engineering the sterile insect technique. Insect Biochem. Mol. Biol. 32: 1243-1247.

Alphey, L., and M. H. Andreasen. 2002. Dominant lethality and insect population control. Mol. Biochem. Parasitol. 121: 173-178.

Alphey, L., D. Nimmo, S. O’Connell, and N. Alphey. 2007. Insect population suppression using engineered insects. In S. Aksoy [ed.], Transgenesis and the management of vector-borne disease. Landes Bioscience, Austin, TX.

Alstad, D. N., and D. A. Andow. 1995. Managing the evolution of insect resistance to transgenic plants. Science (Wash., D.C.) 268: 1894-1896.

Bates, S. L., J. Z. Zhao, A. M. Shelton, and R. T. Roush. 2005. Insect resistance management in GM crops: past, present and future. Nat. Biotechnol. 23: 57-62.

Bloem, K. A., S. Bloem, and J. E. Carpenter. 2005. Impact of moth suppression/eradication programmes using the sterile insect technique or inherited sterility, pp. 677-700. In V. A. Dyck, J. Hendrichs, and A. S. Robinson [eds.], Sterile insect technique. principles and practice in areawide integrated pest management. Springer, The Netherlands.

Bourguet, D., A. Genissel, and M. Raymond. 2000. Insecticide resistance and dominance levels. J. Econ. Entomol. 93: $1588-1595$.

Caprio, M. A. 2001. Source-sink dynamics between transgenic and non-transgenic habitats and their role in the evolution of resistance. J. Economic Entomology. 94: 698-705.

Carrière, Y., and B. Tabashnik. 2001. Reversing insect adaptation to transgenic insecticidal plants. Proc. R. Soc. Lond. B 268: 1475-1480.

Carrière, Y., C. Ellers-Kirk, M. Sisterson, L. Antilla, M. Whitlow, T. J. Dennehy, and B. E. Tabashnik. 2003. Longterm regional suppression of pink bollworm by Bacillus thuringiensis cotton. Proc. Natl. Acad. Sci. U.S.A. 100: 1519-1523.

Dyck, V. A., J. Hendrichs, and A. S. Robinson [eds.]. 2005. Sterile insect technique: principles and practice in areawide integrated pest management. Springer, Amsterdam, The Netherlands.

Ferré, J., and J. Van Rie. 2002. Biochemistry and genetics of insect resistance to Bacillus thuringiensis. Annu. Rev. Entomol. 47: 501-533.

Ferry, N., M. G. Edwards, J. Gatehouse, T. Capell, P. Christou, and A.M.R. Gatehouse. 2006. Transgenic plants for insect pest control: a forward looking scientific perspective. Transgenic Res. 15: 13-19.

Fu, G., K. C. Condon, M. J. Epton, P. Gong, L. Jin, G. C. Condon, N. I. Morrison, T. H. Dafa'alla, and L. Alphey. 2007. Female-specific insect lethality engineered using alternative splicing. Nat. Biotechnol. 25: 353-357.

Glaser, J. A., and S. R. Matten. 2003. Sustainability of insect resistance management strategies for transgenic Bt corn. Biotechnol. Adv. 22: 45-69.

Gong, P., M. Epton, G. Fu, S. Scaife, A. Hiscox, K. Condon, G. Condon, N. Morrison, D. Kelly, T. Dafa'alla, P. Coleman, and L. Alphey. 2005. A dominant lethal genetic system for autocidal control of the Mediterranean fruitfly. Nat. Biotechnol. 23: 453-456.

Gould, F. 1998. Sustainability of transgenic insecticidal cultivars: integrating genetics and ecology. Annu. Rev. Entomol. 43: 701-726.

Gould, F., A. Anderson, A. Jones, D. Sumerford, D. G. Heckel, J. Lopez, S. Micinski, R. Leonard, and M. Laster. 1997. Initial frequency of alleles for resistance to Bacillus thuringiensis toxins in field populations of Heliothis virescens. Proc. Natl. Acad. Sci. U.S.A. 94: 3519-3523.

Heinrich, J., and M. Scott. 2000. A repressible female-specific lethal genetic system for making transgenic insect strains suitable for a sterile-release program. Proc. Natl. Acad. Sci. U.S.A. 97: 8229-8232.

Huang, F., B. R. Leonard, and D. A. Andow. 2007. Sugarcane borer (Lepidoptera: Crambidae) resistance to transgenic Bacillus thuringiensis maize. J. Econ. Entomol. 100: 164171.

James, C. 2006. Global status of commercialized biotech/GM crops: 2006. ISAAA Briefs No. 35. International Service for the Acquisition of Agri-biotech Applications, Ithaca, NY.

Janmaat, A. F., and J. Myers. 2003. Rapid evolution and the cost of resistance to Bacillus thuringiensis in greenhouse populations of cabbage loopers, Trichoplusia ni. Proc. R. Soc. Lond. B 270: 2263-2270.

Knipling, E. 1955. Possibilities of insect control or eradication through use of sexually sterile males. J. Econ. Entomol. 48: 459-462.

Krafsur, E. 1998. Sterile insect technique for suppressing and eradicating insect populations: 55 years and counting. J. Agric. Entomol. 15: 303-317.

Marec, F., L. Neven, A. Robinson, M.J.B. Vreysen, M. Goldsmith, J. Nagaraju, and G. Franz. 2005. Development of genetic sexing strains in Lepidoptera: from traditional to transgenic approaches. J. Econ. Entomol. 98: 248-259.

Marrelli, M. T., C. K. Moreira, D. Kelly, L. Alphey, and M. Jacobs-Lorena. 2006. Mosquito transgenesis: what is the fitness cost? Trends Parasitol. 22: 197-202.

Moreira, L., J. Wang, F. Collins, and M. Jacobs-Lorena. 2004. Fitness of anopheline mosquitoes expressing transgenes that inhibit Plasmodium development. Genetics 166: 1337-1341. 
Peck, S. L., F. Gould, and S. P. Ellner. 1999. Spread of resistance in spatially extended regions of transgenic cotton: implications for management of Heliothis virescens (Lepidoptera: Noctuidae). J. Econ. Entomol. 92: 1-16.

Rendon, P., D. McInnis, D. Lance, and J. Stewart. 2004. Medfly (Diptera: Tephritidae) genetic sexing: large-scale field comparison of males-only and bisexual sterile fly releases in Guatemala. J. Econ. Entomol. 97: 1547-1553.

Schliekelman, P., and F. Gould. 2000. Pest control by the release of insects carrying a female-killing allele on multiple loci. J. Econ. Entomol. 93: 1566-1579.

Shelton, A. M., J. Z. Zhao, and R. T. Roush. 2002. Economic, ecological, food safety, and social consequences of the deployment of Bt transgenic plants. Annu. Rev. Entomol. 47: 845-881.

Sisterson, M. S., L. Antilla, Y. Carrière, C. Ellers-Kirk, and B. E. Tabashnik. 2004. Effects of insect population size on evolution of resistance to transgenic crops. J. Econ. Entomol. 97: 1413-1424.

Stodola, T. J., D. A. Andow, A. R. Hyden, J. L. Hinton, J. J. Roark, L. L. Buschman, P. Porter, and G. B. Cronholm. 2006. Frequency of resistance to Bacillus thuringiensis toxin CrylAb in southern United States corn belt population of European corn borer (Lepidoptera: Crambidae). J. Econ. Entomol. 99: 502-507.
Tabashnik, B. E., F. Gould, and Y. Carrière. 2004. Delaying evolution of insect resistance to transgenic crops by dominance and heritability. J. Evol. Biol. 17: 904-912.

Tabashnik, B. E., T. J. Dennehy, and Y. Carrière. 2005. Delayed resistance to transgenic cotton in pink bollworm. Proc. Natl. Acad. Sci. U.S.A. 102: 15389-15393.

Tabashnik, B. E., A. L. Patin, T. J. Dennehy, Y. B. Liu, Y. Carrière, M. A. Sims, and L. Antilla. 2000. Frequency of resistance to Bacillus thuringiensis in field populations of pink bollworm. Proc. Natl. Acad. Sci. U.S.A. 97: 1298012984.

Tabashnik, B. E., Y. Carrière, T. J. Dennehy, S. Morin, M. S. Sisterson, R. T. Roush, A. M. Shelton, and J. Z. Zhao. 2003. Insect resistance to transgenic Bt crops: lessons from the laboratory and field. J. Econ. Entomol. 96: 1031-1038.

Thomas, D. D., C. A. Donnelly, R. J. Wood, and L. S. Alphey. 2000. Insect population control using a dominant, repressible, lethal genetic system. Science (Wash., D.C.) 287: 2474-2476

Vacher, C., D. Bourguet, F. Rousset, C. Chevillon, and M. E. Hochberg. 2003. Modelling the spatial configuration of refuges for a sustainable of pests: a case study of Bt cotton. J. Evol. Biol. 16: 378-387.

Received 2 March 2007; accepted 1 July 2007. 